\title{
APERTURA Y COMPRENSIÓN DEL MEDITERRÁNEO MERIDIONAL PENINSULAR AL ESPACIO EUROPEO
}

José Hinojosa Montalvo

Universidad de Alicante

\begin{abstract}
SUMARIO
1. La frontera.- 2. Circulación de técnicas y experiencias mercantiles y de navegación.- 3. La circulación de los hombres.- 4. La circulación de culturas. El marco del Islam e Italia.- 5. Agregación económica y cultural al mundo anglogermánico y nondeuropeo. - 6. Agregación cultural al espacio angloeuropeo.- Conclusiones.
\end{abstract}

En el tránsito del Medievo a la Modernidad el viejo "Mare Nostrum", que tantas civilizaciones vio surgir en su regazo, -y también desaparecer en el inexorable curso de la Historia-, era un continuo fluir de gentes de las más variadas condiciones, mercaderes, piratas, soldados, estudiantes, cautivos, clérigos, peregrinos..., y también de productos, desde las exóticas especias al cotidiano cereal; sin olvidar las ideas, el arte o las innovaciones técnicas. Aunque el Atlántico se alzaba pujante y con un prometedor futuro, las aguas de este "gran lago" todavía estaban llenas de vida. Y por mucho tiempo, por fortuna para sus ribereños.

No va a ser nuestro objetivo el Mediterráneo en sí o los diversos mares que alberga, objeto de numerosos trabajos y con la obra clásica de Fernand Braudel presidiéndolos, sino ese canal de la Mancha mediterráneo, como lo calificó el citado historiador francés, un mar sin nombre (el mar 
de Alborán es sólo una pequeña parte del mismo), que irfa desde las proximidades de Argel hasta el cabo de la Nao y el estrecho de Gibraltar'.

¿Cómo se veŕa desde fuera este espacio Mediterráneo?, ¿qué cambios se estaban produciendo en este periodo de transicion de los Tiempos Modernos y cómo se insertaba en ese mundo anglogermánico y nordeuropeo, que tan lejos quedaba desde la perspectiva geográfica?.

Nadie mejor que un viajero centroeuropeo, el alemán Jerónimo Münzer, que en octubre de 1494, en compañfa de sus tres amigos, lleg6 a Alicante, procedente de Valencia "ciudad mucho mayor que Barcelona", según dice textualmente, y le llamó la atención la existencia en su puerto de 26 naves de Vizcaya, de Flandes y de otros paises que iban por cargamento de vino. Y alli se encuentra con un compatriota alemán, el mercader Iodoco Schedler, natural de Kempten, que les depara una cordial acogida, y les explica que ese vino va a Inglaterra, Flandes y otros paises ${ }^{2}$.

Münzer fue un excelente observador, al margen de algunas exageraciones, y sus apreciaciones muestran cómo Valencia era un magnifico observatorio del área mediterránea peninsular. Era la primera vez que un viajero forastero de la Europa del Norte nos dejaba por escrito sus impresiones sobre una pequeña ciudad situada en la periferia europea, Alicante, que para entonces ya estaba plenamente integrada en los circuitos mercantiles europeos de largo alcance, desde Italia a Flandes. Hombres y espacio se fundran en uno gracias al vino, a las pasas, al arroz o a los tejidos y manufacturas norteñas ${ }^{3}$.

No era un fenómeno exclusivo de Alicante, puesto que afectaba también a otras áreas meridionales de la península y del Mediterráneo: al reino de Murcia, a la Andalucía penibética, el viejo reino nazarf, e incluso

\footnotetext{
'Además de la clásica obra de F. BRAUDEL, El Mediterráneo y el mundo mediterráneo en la época de Felipe II, Madrid, 1976, $2^{2}$ edición en español, pueden verse, VV.AA. La Peninsula Ibérica y el Mediterráneo centro-occidental (ss. XII-XV), Barcelona-Roma, 1980; VV.AA. "Actes du Congrès d'Histoire et de Civilisation du Maghreb", Tunis, 1974; VV.AA. La Mediterrània: realitat o inetâfora, Valencia, 1993. VV.AA. 1490: En el umbral de la Modernidad. El Mediterráneo europeo y las ciudades en el tránsito de los siglos XV-XVI, Valencia, 1994. 2 vols.

2J. GARCfA MERCADAl, editor, Viajes de extranjeros por Espana y Portugal, Madrid, 1952. p. 345.

3J. Hinojosa MonTalvo, De Valencia a Portugal y Flandes, "Anales de la Universidad de Alicante. Historia Medieval, 1 (1982), pp. 149-168; El puerto de Alicante durante la Baja Edad Media, "Anales de la Universidad de Alicante. Historia Medieval", 4-5 (1986), pp. 151166.; "La economía", Historia de la Ciudad de Alicante. II Edad Media, Alicante, 1990, pp. 321.354 .
} 
al Norte de Africa, de cultura y religión opuestas, el Islam, pero con viejos y constantes lazos con la otra orilla del Mare Nostrum, unos pacificos, otros, a cargo, de piratas, no tanto.

Contrastando con la vitalidad de Alicante, Münzer se refiere a la decadencia de Cartagena, de la que dice "hoy medio derruida y reducida a la categoría de villa". Por contra destaca la fertilidad de la huerta de Murcia.

Frente a las breves descripciones para el reino de Murcia, se extiende con detalle en el reino de Granada, recién incorporado a la Corona de Castilla. La huella y la presencia musulmana es aqui muy palpable, como también la cercanía del norte de Africa, de Orán y Tremecén, con las que Almería mantiene intensos lazos comerciales y humanos, en un marco espacial perfectamente articulado, en el que catalanes, valencianos y genoveses tenían sus colonias y realizaban sus negocios. No alude a su actividad mercantil nuestro curioso viajero, pero si que se fija en una alta columna situada a la salida de la ciudad, en la que hay colgados por los pies seis italianos acusados de sodomía. Málaga es calificada como famoso puerto de mar, con dos puertos y tres fuertes torres, y se hace eco de la peligrosidad de los desembarcos piratas, conocedores a la perfección del terreno, y del riesgo de caer en cautividad que suponía vivir en aquellas comarcas del litoral granadino.

En el tránsito del Medievo a la Modernidad circulan por el Mediterráneo hombres, ideas y culturas muy variadas, pero con un fondo homogéneo que iba desde la geografía a las formas de poblamiento, desarrollándose en este marco unas formas de agregación más culturales que económicas respecto a los paises atlánticos, y en el que el área valenciana constitufa un observatorio privilegiado. Su situación fronteriza entre la Cristiandad y el Islam desde el siglo XIII habra propiciado la movilidad de la población, el desarrollo de nuevas técnicas mercantiles y de navegación, así como los más variados contactos con el mundo italiano y árabe 4 .

Veamos algunas de estas propuestas en el espacio que se extiende a partir del mediodía valenciano, desde Alicante al Estrecho de Gibraltar,

\footnotetext{
4P. IRADIEl, Prólogo al no 3 de la "Revista d'História Medieval", dedicada a "València i la Mediterrània medieval", Valencia, 1992. p. 8.
} 
dejando aparte a la ciudad de Valencia objeto de otros estudios especifi$\cos ^{5}$.

\section{LA FRONTERA}

Si queremos captar la realidad espacial de este marco geográfico que estamos analizando, el Mediterráneo meridional peninsular, o la Mancha mediterránea, según se prefiera, debemos tener en cuenta un hecho básico que condicionó su trayectoria historica durante toda la Edad Media y buena parte de los tiempos modernos. Me refiero a su condición de frontera, que arranca para las tierras alicantinas y murcianas desde mediados del s. XIII, a partir de su incorporacion a la cristiandad. El tema ha sido muy bien estudiado por M.T. Ferrer Mallol en el ámbito de la gobernación oriolana en el siglo XIV. Una frontera que, como indica dicha autora, era todo el territorio que podía ser amenazado por una razzia granadina de 50 6 días de duración, o por los golpes de manos de los almogávares, ladrones de ganado y secuestradores de personas ${ }^{6}$. La mitad meridional del reino de Valencia era la más amenazada por las expediciones terrestres, que contaban además con el imprevisible peligro de la presencia mudéjar en estas tierras, que podían actuar de quinta columna en apoyo de sus correligionarios.

Los granadinos no fueron la única amenaza contra el espacio y las gentes valencianas o murcianas, sino que en el caso valenciano, las tierras de Alicante fueron también frontera interestatal entre Castilla y Aragón.

\footnotetext{
'A. FURIÓ, editor, Valencia, un mercat medieval, Valencia, 1985; J. GUIRAL-HADZJossIF, Valencia. Puerto mediterráneo en el siglo XV (1410.1525), Valencia, 1989; J. HINOJOSA MONTALVO, Sobre mercaderes extrapeninsulares en la Valencia del siglo XV, "Saitabi", 26 (Valencia, 1976), pp. 62-71; D. IGUAL, Valencia y Sevilla en el sistema económico genovés de finales del siglo XV, "Revista d'Historia Medieval", 3 (Valencia, 1992), pp. 79-116.

'M.T. FERRER I MAWOL, La frontera amb l'Islam en el segle XIV. Cristians $i$ sarralins al País Valencid, Barcelona, 1988. La bibliografía sobre la frontera es muy amplia, y entre la más reciente puede verse la obra VV.AA. La incorporación de Granada a la Corona de Castilla, Gransda, 1993, con diversos estudios, entre ellos el de M. GONZÁLEZ JiMÉNEZ, La frontera entre Andalucía y Granada: realidades bélicas, socioeconómicas y culturales, pp. 87 146, con extensa bibliografía. El 22-24 de noviembre de 1994 se celebró en Lorca-Vera el congreso sobre "La frontera oriental nazari como sujeto histórico (s.XIII-XVI)", cuyas actas están en curso de publicación.
} 
Desde la sentencia de Torrellas, $1304^{7}$, pasaron a ser frontera con el reino de Murcia, con todos los inconvenientes que ello suponía en tiempo de guerra y que fueron una realidad en la guerra de los dos Pedros o en la guerra con Castilla en 1429-1430: robos, cautivos, muertes, destrucción y ruina de la zona ${ }^{8}$. Ello explica la importancia militar que los reyes de Aragón concedieron siempre a estas tierras y a sus fortalezas, consideradas como la clau del Regne, como era el caso de la de Alicante ${ }^{9}$. Los documentos aluden siempre a esta condición de frontera para las plazas de Orihuela o Alicante.

La frontera tenía también su vertiente marítima, no menos importante y decisiva que la terrestre, debido a la proximidad de las costas granadinas y norteafricanas, a la presencia de sus piratas y corsarios, que con sus ataques fueron un serio obstáculo para el desarrollo demográfico y socio-económico del territorio, tanto en tierras alicantinas como murcianas. Pensemos en el despoblamiento y la concentración de la población de núcleos amurallados en el reino de Murcia o de la huerta de Alicante por causa de la inseguridad del territorio, la abundancia de espacios yermos, etc ${ }^{10}$.

Por supuesto que del otro lado, en Granada, nos encontramos con idéntico panorama: correrías, cabalgadas, robos, cautiverios, etc. de sus poblaciones fronteras, en tierra interior o en el litoral, a cargo de almogávares, piratas y corsarios cristianos.

Pero a fines de la Edad Media esta situación va a experimentar importantes cambios, comenzando por la propia frontera, que tras unos

${ }^{7}$ J.M. DEL ESTAL, Conquista y anexión de las tierras de Alicante, Elche, Orihuela y Guardamar al Reino de Valencia por Jaime II de Aragón (1296-1308), Alicante, 1982; M.T. FERRER I MALLOL, Notes sobre la conquesta del regne de Múrcia per Jaume II (1296-1304), "Homenatge a la Memòria del Professor Dr. Emilio Sáez. Aplec d'estudis dels seus deixebles i col.laboradors", Barcelona, 1989, pp. 27-44.

8J. HINOJOSA MONTALVO, Las fronteras valencianas durante la fuerra con Castilla (14291430), "Saitabi", XXXVII (1987), pp. 149-157; Crónica de Pere Maça de Liçana, Valencia, 1979.

'J. Hinojosa Montalvo, La clau del regne, Alicante, 1990.

${ }^{10} \mathrm{M}$. SANCHEZ MARTINEZ, Comercio nazañ y piraterla catalano-aragonesa (1344-1345), "Relaciones de la Península Ibérica con el Magreb. Siglos XIII-XVI", Madrid, 1988, pp. 4175; A. DIAZ BORRÁs, Los orfgenes de la piratería islámica en Valencia. La ofensiva musulmana trecentista y la reacción cristiana, Barcelona, 1993; J. HinoJosa MONTALVO, La piratería y el corso en las costas alicantinas durante la Baja Edad Media, "VIII Jomades d'Estudis Històrics Locals. El comerç alternatiu. Corsarisme i contrabán (ss. XV-XVIII)", Palma de Mallorea, 1990, pp. 41-54. 
años de movilidad desaparecerá al incorporarse a la cristiandad el reino de Granada. El peligro granadino ya no existirá, como también habra dejado de existir el riesgo de guerra en la frontera valenciano-murciana tras el matrimonio de Fernando e Isabel. La paz, la seguridad del territorio y de sus gentes, marcará el devenir histórico en el futuro y permitirá la reactivación económica de muchas comarcas, a la vez que destierra la vida de fronteras que hasta ahora había caracterizado esta sociedad.

No sucedio lo mismo con la frontera marítima, pues si bien desapareció la piratería granadina, hubo un espectacular incremento de la berberisca, hasta el punto de que las alquerias y viviendas dispersas de la huerta alicantina hubieron de fortificarse y todo el litoral mediterráneo desde el Ebro al estrecho de Gibraltar se cuajo de torres vigfa ${ }^{11}$.

Más al oeste, el fondo del Mediterráneo, ese cul de sac donde entra en contacto con el Atlántico, el Estrecho de Gibraltar, fue durante los siglos medievales también una frontera. Si hasta mediados del siglo XIV, la Andalucía cristiana, integrada en la Corona de Castilla, estuvo frecuentemente a la defensiva, a partir de la batalla del Estrecho hay un claro cambio en el signo de fuerzas, de marcado predominio cristiano.

La descomposición del poderfo merinf en Fez trajo también para el norte de Marruecos un claro desplazamiento del ámbito de intereses y contactos, de la tierra al mar. Un mar dominado por los barcos cristianos. En el s. XV ya no hay restos de la batalla del Estrecho, sino un proceso de conquista de las rutas marftimas en el Atlántico medio y de enclaves costeros norteafricanos, por las marinas de Castilla y de Portugal. Para los hombres contemporáneos del espacio mediterráneo meridional el señuelo de la guerra contra el infiel y los proyectos de conquista en su territorio estaban fuertemente arraigados en su mentalidad. El emirato de Fez quedó reservado a Portugal, y la conquista de plazas litorales constituyo un capítulo muy importante en los esfuerzos de expansión atlántica lusitana durante el $\mathbf{s}$.

\footnotetext{
"J. GARCtA ANTON, La costa de Lorca y la frontera martrima, "Lorca, pasado y presente", I, Lorca, 1990; M. MARTINEZ MARTINEZ, La frontera murciano-granadina en la Baja Edad Media, "Nuestra Historia", Cartagena, 1987, pp. 129-150; F.J. SElJO AlONSO, Torres de vigia y defensa del Reino de Valencia, Alicante, 1978; F.J. JOVER MAESTRE y J.L. MENENDEZ FUEYO, Las torres de costa de la bahía de Alicante: una visión arqueologica, "Castells", 4 (Alicante, diciembre 1994), pp. 30-37.
} 
XV. Recordemos en el área del Estrecho la conquista de Ceuta (1415), Alcazarquivir (1458), o Tanger en 1471, Arcila, etc ${ }^{12}$.

Lo habitual era que a finales del s. XV los marinos andaluces atacaran las costas de Berberfa, de Fez y Tremecén, mediante cabalgadas que reportaban botín y cautivos. Eran empresas arriesgadas y lucrativas, que servían también para aumentar el conocimiento detallado de la costa enemiga y contar con expertos útiles, llegado el caso. Que no tardo.

Naturalmente, los musulmanes replicaban, en la medida de sus fuerzas, y éstas aumentaron desde el segundo decenio del siglo XVI, con el auge e la piratería berberisca y la desviación del esfuerzo naval y militar castellano hacia otros ámbitos considerados más importantes. Las localidades costeras saqueadas por los piratas y corsarios en este periodo abarcan todo el arco sur mediterráneo, desde Cullera a Conil, con un balance negativo para sus gentes. La multitud de torres que jalonan todo el litoral, desde Málaga a Castellón, son el mejor testimonio del peligro que se cernió sobre esta "frontera olvidada", como la calificó A.C. Hees ${ }^{13}$, y que mantuvo una imagen negativa, acrecentando la enemistad hacia "el otro", hacia el infiel, consecuencia también de la colaboración de los moriscos valencianos o granadinos como quinta columna de turcos y piratas. Una brecha insalvable se abría entre ambas comunidades y creencias ${ }^{14}$.

La conquista del reino de Granada supuso para la corona de Castilla la incorporación de la manga mediterránea, la que va desde Palos al Estrecho de Gibraltar, una nueva y extensa fachada maritima, un nuevo espacio maritimo, con todo lo que ello implicaba. No es que Castilla hubiera estado ausente del Mediterráneo, puesto que la conquista del reino de Murcia le proporcionó una ventana a este mar, a través de los puertos de Cartagena y de Alicante, éste luego incorporado al reino de Valencia. Pero Cartagena servía sólo al traspaís murciano y manchego. El resto de Castilla basculaba y miraba hacia el Atlántico, en sus dos fachadas la cantábrica y la meridional. Pero desde mediados del siglo XIV y la apertura del Estre-

12" Actas del Congreso Intemacional 'El Estrecho de Gibraltar'", Céuta, 1987. Edición por E. Ripoll Perello, Madrid, 1988.

${ }^{13}$ A.C. HEES, The Forgotten Frontier. A History of the Sixteenth-Century Ibero-African Frontier, Chicago, 1978. M.A. LADERO QUESADA, Castilla, Gibraltar y Berbería (12521516), "Congreso Intemacional. El Estrecho de Gibraltar, Ceuta, 1987, II, Madrid, 1988, pp. $37-64$.

${ }^{14}$ Actas del Coloquio: "Relaciones de la Península Ibérica con el Magreb. Siglos XIIIXIV", (Madrid, 1987), Madrid, 1988. 
cho de Gibraltar, el Mediterráneo dejo de ser un mar cerrado y el flujo de hombres, barcos, mercancías e ideas entre el Mediterráneo y el Atlántico fue una realidad que crecía día a día, sobre todo desde finales del siglo XIV. En el Cuatrocientos, Castilla está en el Mediterráneo, con sus barcos, sus mercaderes y sus piratas, pero no es una potencia mediterránea. La conquista de Granada le hará asumir este nuevo papel y la imbricará directamente con el Norte de Africa, visto como una prolongación de las costas andaluzas, como un área de expansión que hay que dominar.

Desde 1492 Granada deja de ser frontera terrestre y marítima, pero las relaciones y contactos con el Norte de Africa continuaron. Hubo estrategas y políticos que consideraron las posibilidades y la conveniencia de prolongar la expansión castellana al otro lado del Mar de Alborán, sobre las costas del emirato de Tremecén, ya que las de Fez correspondian al ámbito de influencia portuguesa. La ocupación de diversas plazas y presidios a fines del siglo XV y comienzos del XVI: Melilla, Cazaza, Mazalquivir, Velez de la gomera, Orán, etc. trasladó la frontera allende el mar y origino intensos cambios en el espacio terminal del Mediterráneo, desde movimientos de población voluntarios o forzosos (cautivos de ambas partes) a importantes novedades en la actividad corsaria del Estrecho, que si en el siglo XV la controlaban los portugueses y castellanos, a partir de la fundación granadina de Tetuán van a ser de signo claramente granadino, alcanzando una virulencia extraordinaria y convirtiendo el mar de Alborán en una zona peligrosa para el tráfico marítimo y las costas hispanas se verán continuamente asaltadas por estos corsarios berberiscos, como se les conoce en la historiografía, pero que en realidad son moriscos hispanos que han convertido el mar en una prolongación de la lucha terrestre, perdida por ellos en la península ${ }^{15}$.

Todos estos cambios geoestratégicos en el espacio meridional del Mediterráneo, el llevar la frontera allende, tuvieron importantes repercusiones económicas en toda el área. El abastecimiento de Melilla, de Cazaza y los demás enclaves se realizo desde los puertos atlánticos andaluces, pero también desde Málaga, lo que nos lleva a plantear otra cuestion de interés que ha sido objeto de investigaciones recientes: la de cómo se reanudo el comercio entre Granada y Berbería después de la incorporación de aquel

\footnotetext{
15J.E. LÓPEZ DE COCA CASTAÑER, Granada y el Magreb: la emigración andalusi (14851516), "Relaciones de la Península Ibérica con el Magreb (s. XIII-XVI", Madrid, 1988, pp. $409-452$.
} 
reino a la Corona de Castilla, bien estudiado por E. Lopez de Coca y M.T. Lopez Beltrán ${ }^{16}$. Vemos como a petición del concejo de Málaga, tanto el papa como los reyes concedieron permiso para el tráfico con Berbería, salvando las habituales prohibiciones de productos vedados. En 1491 recibieron permisos semejantes Almería y Marbella, pero Málaga conservó la posición principal que habra tenido en epoca nazari, tanto en el comercio con Tremecén como en las escalas que los barcos venecianos y genoveses hacían en su puerto, procedentes de Orán y otras plazas norteafricanas. Un tráfico peculiar se estableció con Vélez de la Gomera, basado en el rescate de cautivos, sin olvidar la función informativa que se derivaba de aquellos tráficos navales ${ }^{17}$.

En resumen, hacia 1515 este proceso de expansión y dominio de los marinos castellanos había alcanzado su apogeo y habia dado lugar, también, a una situación estretágica de claro predominio en la zona y a unos hábitos de comunicación hostil o comercial con el Magreb que perdurarfan largo tiempo. El dominio militar castellano alejaba la preocupación por el control del Estrecho, pero el peligro de la piratería musulmana fue una amenaza convertida en realidad en el Quinientos ${ }^{18}$.

\section{Circulación de técnicas y EXPERIENCIAS MERCANTILES Y DE NAVEGACIÓN}

En esta Mancha mediterránea a fines de la Edad Media circulan hombres, mercancias y técnicas, que ya no son las mismas que en los siglos anteriores y que preludian a las de los tiempos modernos.

Tomando como observatorio, en primer lugar, la villa de Alicante lo primero que llama la atención, no sólo al viajero alemán sino también al historiador, es el papel clave que el puerto ha jugado a lo largo de su histo-

\footnotetext{
${ }^{16} \mathrm{~J} . \mathrm{E}$. LOPEZ DE COCA, Relaciones mercantiles entre Granada y Berbería en la época de los Reyes Católicos, "Baietica", 1 (1978), pp. 296-298. En particular. F. BEJARANO ROBLES, Reanudación del comercio de Málaga con Africa bajo los Reyes Católicos, "Homenaje a Guillermo Gustavino", Madrid, 1974, pp. 429-441; M. Teresa LOPEZ BELTRAN, El puerto de Málaga en la transición a los tiempos Modemos, Málaga, 1986.

${ }^{17}$ J.E. LOPEZ DE COCA, Vélez de la gomera y su puerto durante la primera mitad del siglo XVI, "Historia. Instituciones. Documentos", 20 (1993), pp. 207-230.

${ }^{18}$ M.A. LADERO QUESADA, Castilla, Gibraltar y Berbería (1252-1516, "Los mudéjares de Castilla y otros estudios de historia medieval andaluza", Granada, 1989, pp. 169-219.
} 
ria. Ya Alfonso el Sabio le concedio el privilegio de comerciar con Ultramar, con el Norte de Africa, y a fines del Medievo prácticamente era el único puerto que podía calificarse como tal en el reino de Valencia, con su muelle de piedra de 200 pasos de longitud, y el segundo en volumen de tráfico, tras el de Valencia.

La novedad en estos años desde el punto de vista de las técnicas y el arte de la navegación es que el comercio de larga distancia aparece monopolizado por naves y carabelas, estas últimas de andaluces y portugueses. En el comercio entre Alicante y Flandes, por ejemplo, se produjeron importantes modificaciones en el transporte entre 1468 y 1490 . Si en el primero de estos años predominan las carabelas, galeras y galeazas (éstas, florentinas y venecianas hacen la ruta Italia-Flandes), antes de 1485 han desaparecido estas dos últimas embarcaciones en la ruta de Flandes y en 1490 el monopolio de la nave es total. Más tarde, en las primeras décadas del Quinientos veremos similar fenomeno en Venecia, que abandona sus tradicionales galeras por las naves de alto porte. En el comercio de cabotaje hay un mayor apego por la tradición y se siguio con los tradicionales laudes, balleneros, leños y barcas. También en Málaga se observa idéntico predominio de la nao y la carabela sobre el resto de embarcaciones en la navegacion internacional.

El comercio en este espacio mediterráneo estaba en manos de mercaderes locales, pero la gran novedad es la afluencia masiva de castellanos, sobre todo vascos y andaluces, que actúan como transportistas, marineros y abastecedores de pescados ${ }^{19}$.

Es diff́cil, por la falta de fuentes, valorar el volumen y la importancia que pudo tener el comercio entre Alicante y el Norte de Africa a fines de la Edad Media, pero a tenor de lo que sucedía en Valencia, con un claro retroceso, no parece que fuera muy importante. Todos los datos apuntan a que Alicante mira sobre todo hacia el Norte de las costas mediterráneas peninsulares, las islas mediterráneas e Italia. Los intercambios con Africa y Granada debieron ser esporádicos y marginales. La instalación de los presidios españoles en el Magreb reactivará este comercio y Alicante se convertirá en uno de los centros abastecedores de Orán, Mazalquivir, etc. En la ciudad de Valencia la recuperacion se produjo en 1509-1510 cuando el

\footnotetext{
${ }^{19} \mathrm{~J}$. HinOJOSA MONTALVO, Alicante, polo de crecimiento en el tránsito de los siglos XV al XVI, "1490: en el umbral de la Modernidad. El Mediterráneo europeo y las ciudades en el tránsito de los siglos XV al XVI", I, Valencia, 1994, pp. 71-108.
} 
converso y lugarteniente del tesorero del rey, Alfonso Sánchez, obtiene una concesión real para comerciar con el Norte de Africa, pero a partir de 1519-20, abandonada la conquista de Africa la caida del tráfico con Berbería es total ${ }^{20}$.

Respecto al reino de Murcia, Castilla se abre al Mediterráneo por el puerto de Cartagena, base mercantil, de escala en las rutas mediterráneas y atlánticas, y refugio de piratas y corsarios. Pero en el reino faltan hombres de negocio al estilo de un Pere Monsalve, de principios del s. XV, estudiado por Benito Ruano ${ }^{21}$. Ello provoca la necesidad de mercaderes extranjeros, sobre todo genoveses, por sus dinero y sus mercancías.

Como señala Torres Fontes el renacer económico de Murcia está vinculado a la presencia de estos extranjeros, que participan activamente en la importación y la industria del tinte, del pastel y del alumbre, el negocio más rentable para los italianos en estas tierras. La importación de toda clase de mercaderf́as, exportación de productos del país fue el próspero negocio al que los mercaderes italianos dedicaron su esfuerzo, capital y experiencia. A partir de 1492 los genoveses se hicieron indispensables al convertirse en los únicos con el potencial economico necesario para efectuar negocios importantes, incluso como prestamistas del concejo ${ }^{22}$. En cambio, en Cartagena, en el Quinientos predominan los naturales en la actividad mercantil, y la penetración de los genoveses fue lenta ${ }^{23}$.

Dentro de la circulación de experiencias estos italianos, al igual que en Valencia con la seda o el papel, iniciaron también nuevas industrias o métodos innovadores, que mantuvieron en tanto fueron productivos o no hubo competencia castellana, como la del tinte en Murcia. Sin olvidar, claro está, la difusión de la imprenta y el papel técnico de los alemanes en ciudades como Valencia o Murcia.

\footnotetext{
${ }^{20}$ A. DIAZ BORRAs, La casa de contratación de Orán y el cambio en la filosofía de las transacciones entre Berberia y Valencia. 1510-1514, "Sharq al-Andalus", 9 (1992), pp. 19-28.

${ }^{21}$ E. BENITO RuANO, Avisos y negocios del mercader Pere de Monsalve, "Boletín de la Real Academia de la Historia", CLXIX (1972), pp. 139-169.

22J. TORRES FONTES y A. Luis MOUNA MOLNA, El adelantamiento murciano, marca medieval de Castilla, "Historia de la Región Murciana", IV, Murcia, 1980, pp. 87-92.

${ }^{23} \mathrm{~V}$. Montojo Montojo, Cartagena en la época de Carlos V, Murcia, 1987; A.L. Mouna Mouna, Proyección mediterránea del Reino de Murcia en la Edad Media, "Miscelánea Medieval Murciana" XVII (1992), pp. 59-75; Mercaderes genoveses en Murcia durante la época de los Reyes Católicos (1475-1516), "Miscelánea Medieval Murciana", II (1976), pp. 277-312.
} 
En Málaga, la singularidad de los factores geográficos (proximidad a los puertos norteafricanos y su situación privilegiada en la ruta del Estrecho) le permiten, tras la conquista cristiana, continuar desempeñando la función de cabeza de puente del tráfico con el norte de Africa y escala en los navíos que hacen la ruta del Estrecho. Sin olvidar, como recuerda Lopez de Coca, su papel de puerto de entrada y salida para los productos procedentes o destinados a la Andalucía media y alta, que hacen de Málaga un mercado lleno de atractivos para la clase mercantil, aunque carezca de un muelle que ofrezca un refugio seguro a los navios que alls arriban.

La colonia extranjera más importante es la genovesa, herencia de la época nazari, y son los grandes protagonistas del comercio exterior malagueño, sin una especialización clara, y con un interés más hacia la Andalucía media que hacia el Norte de Africa, donde su papel se ha malogrado's.

\section{LA CIRCULACIÓN DE LOS HOMBRES}

Un aspecto que no conviene olvidar en el marco espacial de ambas orillas del Mediterráneo es la circulación humana, que es tan vieja como el hombre. Recordemos que durante buena parte del s. XIII y aún en el XIV, hubo grupos de mercenarios cristianos (catalanes, valencianos o mallorquines, castellanos) al servicio de los sultanes norteafricanos. En el siglo XV parece que cesa por completo esta presencia de grupos armados cristianos de origen foráneo en los sultanatos magrebres, aunque continuo, al menos, en proyecto, la organización de misiones evangelizadoras en el Africa islámica: recordemos que hay un obispo de Marruecos, franciscano con sede en Sevilla, durante la baja Edad Media.

También hay que mencionar la circulación de viajeros y peregrinos musulmanes que se mueven por ambas orillas de este espacio mediterráneo, llevando y trayendo noticias, ideas, difundiendo el bagaje cultural dentro

\footnotetext{
${ }^{24} \mathrm{~J}$.E. LOPEZ DE COCA, Málaga, "colonia" genovesa (siglos XIV-XV), "Cuademos de Estudios Medievales", 1 (Granada, 1973), pp. 135-144; y M.T. LOPEZ BELTRAN, Mercaderes genoveses en Málaga (1487-1516). los hermanos Centurión e Ytalian, "Historia. Instituciones. Documentos, (Sevilla, 1981), pp. 1-29; J.E. LOPEZ DE COCA, Los genoveses en Málaga durante el reinado de los Reyes Católicos, "Actas del I Congreso Intemacional de Historia Mediterránea" (Palma de Mallorca, 1973), en "Anuario de Estudios Medievales", 10 (1980), pp. 619-650.
} 
del área islámica, aunque cronológicamente comprende hasta la época anterior a la caida de Granada ${ }^{25}$.

Junto a los mercaderes que se mueven entre ambas costas del Mediterráneo merece destacarse un colectivo singular y abundante en estos años a caballo entre el mundo medieval y moderno, cual es el de los cautivos, cristianos o musulmanes, desde Valencia y Murcia a los sultanatos magrebres, pasando por el corral de Granada. El cautivo es un personaje consustancial a la sociedad bajomedieval, que genera una compleja casuística, desde las ordenes redentoras a personajes especializados en intercambiarlos. Abundan en las ciudades del Mediterráneo occidental y generan una auténtica industria de la guerra vinculada al corso. Valencia y Alicante son a finales del siglo XV los dos puertos de venta de esclavos más activos del Mediterráneo peninsular, a su vez estrechamente conectados con hombres y capitales en otras ciudades, como Sevilla y Lisboa, y con los mercaderes italianos y castellanos que actúan como agentes intermediarios. Esclavos aficanos son traidos por mercaderes florentinos desde Sevilla a Valencia y reexpedidos a Lisboa, en un circuito en el que se funden la circulación de hombres y de mercancías ${ }^{26}$.

La calda de Granada produjo un importante cambio en la circulación de cautivos, al cesar el tráfico forzoso de cautivos cristianos y nazaríes. El cautivo granadino será sustituido en Valencia y otras plazas por el canario y el negro, mientras perdura el esclavo norteafricano ${ }^{27}$. En cambio, el valenciano, el murciano y el andaluz penibético seguirán siendo cautivos del Islam, no del granadino, sino del Norteafricano. Las correrfas de piratas y corsarios berberiscos llenaron las cárceles de Argel de cristianos.

En el capítulo de la circulación de las personas en el tránsito a la Modernidad no debemos olvidar un aspecto muy peculiar de nuestra penín-

\footnotetext{
${ }^{25}$ María Lutgarda ORTELIS, Los mudéjares de Valencia en el siglo XV a través de los Delmaments de Sarrains, "Actas del V Simposio Intermacional de Mudejarismo", Teruel, 1991, pp. 135-146.

${ }^{26} \mathrm{~J}$. Hinojosa MONTALVO, Cesaro Barchi y otros mercaderes florentinos en la ciudad de Valencia en el tránsito del Medievo a la Modernidad, "Sardegna, Mediterraneo e Atlantico tra Medioevo ed Età Modema. Studi Storici in Memoriam di Alberto Boscolo", III, Roma, 1993, pp. 231-249.

${ }^{27} \mathrm{~V}$. CORTÉs, La esclavitud en valencia durante el reinado de los Reyes Católicos, Valencia, 1964; J. Hinojosa MONTALVo, La esclavitud en Alicante a fines de la Edad Media, "Les sociétés urbaines dans la France méridionale et la Péninsule lbérique" (Pau, 1988), Paris, 1991, pp. 373-392.
} 
sula, cual fue el de los emigrantes a la fuerza y desterrados: los granadinos que marcharon a Marruecos tras la caida de su reino ${ }^{28}$ y la diaspora sefard tras el decreto de expulsión de los Reyes Católicos en 1492. Aunque parte de nuestros judíos marcharon hacia la península italiana y el imperio otomano, importantes contingentes se instalaron en el Norte de Africa, y hubo valencianos que fueron a Argel, Orán o Tremecén, mientras que muchos judíos castellanos preferian las tierras marroquies, como ha estudiado E. Cantera, embarcándose por los puertos de la costa oriental (Cartagena los del reino de Murcia, Almería, Málaga) y meridional de la Península ${ }^{29}$. El judarsmo magrebr iba a verse profundamente alterado por la llegada de estos contingentes de judíos castellanos, que darán lugar a una profunda reorganización de las comunidades autóctonas y a una manifiesta revitalización de su vida cultural y religiosa.

Para hacernos una idea de la importancia de esta aportación judfa de Sefarad en Marruecos basta fijarse, como ha hecho Abraham Laredo, en que la mitad de los cerca de 1.200 apellidos de judíos marroquies son de origen hispano ${ }^{30}$. Es el mejor ejemplo de la circulación de hombres en este espacio mediterráneo.

Sobre la trascendencia que la emigración granadina tuvo en todos los ordenes de vida de Marruecos no hace falta insistir, abarcando un amplio marco que va desde la fundación de Tetuán a las profundas influencias que la cultura nazar! tuvo en el Magreb.

\section{LA CIRCULACIÓN DE CULTURAS El MARCO DEL ISLAM E ITALIA}

El área de la Mancha mediterránea fue también en esta época una circulación de culturas entre ambas orillas del Mediterráneo, con una neta superioridad de la orilla norte sobre la meridional. En el siglo XV, la zona

\footnotetext{
${ }^{28}$ J.E. LOPEZ DE COCA CASTAÑER, Granado y el Magreb: la emigración andalust (14851516), "Relaciones de la Península Ibérica con el Magreb (s. XIII-XVI)", Madrid, 1988, pp. 409-452.

${ }^{29}$ E. CANTERA MONTENEgRo, 31 de marzo: la expulsión de los judios, "Medievalia", 3 (1993), pp. 279-290.

${ }^{30}$ A.I. LAREDO, Les noms de juifs du Maroc, Madrid, 1978.
} 
de Marruecos era una auténtica colonia cultural de Granada en todos los órdenes, desde el teológico al artístico, papel que se vería acrecentado con la llegada de los emigrantes granadinos a partir de 1492 en el terreno de las letras y del arte. En la música, por ejemplo, hay que destacar el transvase del repertorio musical nazarf al Norte de Africa y otros puntos del mundo islámico. Por ejemplo, la Nuba, que surge en Granada y alcanza su forma definitiva emigrando al $\mathbf{N}$. de Africa, reforzando los sustratos culturales que se han ido formando a lo largo del medievo. En Granada, en cambio, aparte de la perduración de su propia música tradicional entre los moriscos, la agrupación renacentista se impuso. Esta huella cultural perdura hasta nuestros días, y viendo, por ejemplo las tumbas saadianas o las mezquitas de Fez el viajero cree recrear el ambiente de la Alhambra o del Alcázar sevillano.

Qué aportó èl Magreb al reino nazarı de Granada culturalmente? Puede decirse que nada. Quizás las respuestas, fatwas, dadas por algunos ulemas magrebres a las consultas hechas por sus correligionarios granadinos, aunque los datos son de principios del siglo XV.

Y lo mismo se puede decir de la aportación cultural tan extraordinaria que los sefardíes realizaron sobre las comunidades hebreas del Magreb, cuyos niveles intelectuales no resistían comparación con los hispanos .Asi, el folklore marroqui se enriqueció desde finales del siglo XV con un buen número de leyendas y narraciones acerca del viaje y asentamiento de los judíos hispanos en diferentes poblaciones marroquies.

La llegada de los sefardíes al Magreb provocó una alteración sustancial en la vida de las comunidades hebreas autóctonas, como ha estudiado Haim Safrani, comenzando por la lengua, que en el primer momento creб una barrera entre judfos inmigrantes y autoctonos, y la manifiesta superioridad intelectual y teologica de los sefardfes, que provocó el recelo de los rabinos de las comunidades autóctonas, que temían perder el control del judaísmo magrebi, como sucedio a la larga ${ }^{31}$.

El proceso de unificación varió según los lugares y en las grandes ciudades como Fez, Mequinez, Orán, Argel o Tremecén, los sefardíes no fueron más que una minoría y el judaísmo hispánico se diluyó paulatinamente, a pesar de que su tradicion intelectual no tard 6 en imponerse. En

\footnotetext{
${ }^{31} \mathrm{H}$. SAFRANI, Mille ans de vie juive au Maroc. Histoire et culture, religion et magie, Paris, 1983.
} 
cambio, en el norte de Marruecos: Tetuán, Tanger, Larache, etc. la lengua castellana y las tradiciones sefardíes se impusieron rápidamente en el conjunto de la comunidad judía, prevaleciendo hasta tiempos recientes. Estas poblaciones se transformaron en el centro vivo y activo del hispanismo magrebi, y es en ellas donde la antigua civilización andalusí se ha conservado más pura de toda influencia extraña.

Los rabinos sefardfes organizaron las instituciones comunales y religiosas, de acuerdo con las normas y costumbres que regían en las aljamas castellanas. La indiscutible superioridad intelectual del judarsmo hispano -visible en las escuelas y sinagogas- dio lugar a un importante renacimiento cultural del judaísmo magrebl, que se abre a las grandes corrientes del pensamiento judío occidental: la obra de Maimónides se impone como recurso último en casos de conflicto en la interpretación de los textos doctrinales, y se difunden las obras de los grandes autores judeo-españoles como Maimónides, Juda Ha-Levi o Ibn Gabirol.

De este modo, y gracias a la circulación de los hombres, aunque en este caso forzosa, a la aportación hispana, el judarsmo norteafricano comenzaba a salir paulatinamente del estancamiento cultural en que se hallaba inmersa desde hacía varias centurias: las academias de Fez recobraron su pasado esplendor y surgieron nuevos focos de gran vigor en Mequinez, Tetuán y Sale. El papel de estos judíos sefardíes como intérpretes o diplomáticos, cuando las plazas del norte de Africa, Bugfa y Orán, pasan a manos españolas, fue esencial. Pero siempre dentro del marco del espacio mediterráneo en el que siempre se habran movido estas comunidades. Cambiaron de orilla, de señor, pero no de espacio. No miraron hacia la Europa anglogermánica como hicieron otros correligionarios peninsulares, por ejemplo los portugueses.

Mucha mayor trascendencia tuvo para la orilla septentrional de esta área mediterránea los contactos culturales con Italia, que son los que marcaron su impronta y dejaron su huella en nuestra cultura. Ni Valencia ni Murcia, a pesar de contar con población mudéjar, es tierra de arte mudéjar. Hay que buscarlo con lupa. En cambio, la pintura italiana está presente por doquier. Y no se trata sólo de pintura, sino que hoy es unánimemente reconocido que el renacimiento tiene su puerta de entrada por Valencia, aunque Ximo Company habla de "Renacimientos" y destaca que la ciudad se erigió como el núcleo más importante y precoz de la Península en su recepción y 
asimilación ${ }^{32}$. Pero los ritmos en las distintas artes y áreas mediterráneas son distintos según los países, y frente a la precocidad de la pintura valenciana, en cambio la arquitectura valenciana hasta bien entrada la primera mitad del siglo XVI, e incluso con posterioridad, apenas supera las adjetivaciones y añadidos renacentistas en unos cuantos edificios de incuestionable estructura medieval. Entre Valencia e Italia hay diferencias cronologicas de casi cien años. Y en escultura, al margen de Damia Forment, la mayor parte de la escultura valenciana del Renacimiento se mueve entre el anonimato y la importación.

Las tierras alicantinas del Sur se desmarcan del resto del reino en el terreno artístico, y la influencia murciana, otro de los focos del temprano renacimiento peninsular, dará lugar a un brote temprano de arquitectura renacentista en Biar y Villena. No olvidemos que la gobernación oriolana pertenecia al obispado de Cartagena. Pero en general el arte permanecia anclado aún en el gótico medieval. De la escasa tradición que todavía tenía el Renacimiento en las tierras alicantinas baste decir que dos obras de gran belleza, las pilas bautismales de Santa María y la de la Santa Faz (1506, desaparecida) eran obras de importación italiana, de los talleres de Carrara. La presencia del renacimiento será, por tanto, más tardía que en la ciudad de Valencia.

El caso de Murcia y las tierras vecinas del reino granadino recién conquistadas tiene sus peculiaridades, con un primer proceso, que es el que nos interesa, de implantación del renacimiento con plena vinculación a las corrientes internacionales. Llama la atención el temprano arraigo del Renacimiento (castillo de los Vélez, 1506, que también se relaciona con el castillo de la Calahorra iniciado en 1509), a lo que contribuyó la voluntad de los patronos y artistas y la falta de unas tradiciones medievales. La presencia del gótico fue escasa y la arquitectura camino por dos vertientes: los grandes monumentos renacentistas (castillo de los Vélez, la torre de la catedral de Murcia, puerta de las Cadenas, que tuvo como fin ampliar y concluir el edificio, darle un rostro monumental y preeminente en la vida urbana, etc), un arte oficial, y las obras vinculadas a la herencia morisca, más afincadas en el medio popular. Señalemos que la corriente renacentista se

\footnotetext{
${ }^{32}$ X. COMPANY y F.V. GARIN LLOMBART, Valencia y la pintura flamenca, en Historia del Arte Valenciano, II. El Gótico, Valencia, 1988; X. COMPANY, La pintura hispanoflamenca (al Pais Valencid), Valencia, 1990; L'art $i$ els artistes al País Valencid modern (1440-1600), Barcelona, 1991.
} 
mantuvo dentro del clasicismo ortodoxo, siguiendo los modelos toscanos y romanos.

También la escultura se halla muy vinculada a los patronos y sensibilidad italianos, sobre todo la producción florentina de fines del siglo XV, aunque también se percibe la huella hispanoflamenca (expresión de los rostros, pliegues y telas) a través de la escuela castellana, pero es muy débil.

En la pintura la influencia y la huella italiana es rotunda, debido a la presencia temprana en Murcia de Hernando de Llanos, que significó la apertura hacia la influencia italiana y la manera de trabajar de Leonardo, asf como un estímulo para los pintores locales ${ }^{33}$.

Desde el punto de vista de las letras, la consolidación política y económica del reino de Valencia se tradujo en un fuerte empuje literario qu corrió paralelo a la renovación de la prosa cancelleresca, iniciada hacia 1381. Los contactos culturales con la Galia (París, Aviñon) permitieron la divulgación de las obras de los primeros humanistas italianos.

La etapa de 1425-1497 será la etapa de plenitud del humanismo literario en Valencia, si bien el humanismo en latín no triunfará hasta bien entrado el siglo XVI, con la obra de Lluís Vives (1492-1540). Es la etapa dorada de la literatura valenciana. En cambio, en el periodo que estudiamos, entre 1497-1523, se puede hablar de un renacimiento frustrado, y como señala A. Ferrando, la etapa se caracteriza por la defección literaria de la aristocracia, por la pérdida de confianza en la utilidad del idiona, por la ausencia de escritores de relieve, por la vitalidad de una producción burguesa de corto vuelo y temática restringida, y por el debilitamiento de la conciencia de la unidad literaria de la lengua ${ }^{34}$.

También dentro de la órbita de la cultura merece reseñarse la presencia de valencianos estudiando leyes en la Universidad de Bolonia o de medicina en las de Siena, Pisa y Ferrara en los finales del siglo XV y comienzos del XVI, testimonio del indiscutible prestigio que gozaban entonces las universidades italianas, y de cómo la presencia de los papas valencianos en Roma, el dominio sobre Nápoles y la consolidación del asentamiento español en Italia hizo que los hispanos, sobre todo los valencianos, se difundieran por todo el país.

\footnotetext{
${ }^{33} \mathrm{C}$. BeIDA NAVARRO, El arte cristiano medieval en Murcia, en Historia de la región murciana, IV, PP. 220-349.

"A. FerRando, Lengua i literatura, en Histzria del País Valencià. De la conquesta a la federacio hispdnica, II, Barcelona, 1989, pp. 379-424.
} 
Todo ello, una vez más, referido a la capital del reino porque de las comarcas meridionales es muy poco o nada lo que sabemos. Ni siquiera lo que lefan las gentes de Elche o Alicante, pero todo apunta a un estadio cultural muy elemental, con excepciones quizá en las oligarquías urbanas. En Orihuela, la capital de la gobernación, la cultura era patrimonio de una minoria, y quien quisiera triunfar debra marchar fuera, como fue el caso de Lope de Espejo, que ya en 1453 residía en Nápoles, donde destacó por su arte de componer versos latinos, escribiendo siempre en latín e italiano. Un estudio de los incunables de la biblioteca catedralicia, quizá el foco intelectual oriolano más potente, podria arrojar más luz sobre la penetración y difusión del humanismo en estas tierras.

En la cercana Murcia las noticias sobre libros, libreros y lectores apuntan a que se lee poco, y fuera de Murcia no hay libreros, y ninguna noticia conozco sobre autores murcianos en estos años de transición.

\section{AgRegación ECONÓMICA Y CULTURAL AL MUNDO GERMÁNICO Y NORDEUROPEO}

Ya vimos como el área meridional del Mediterráneo tuvo poco interés para el comercio alicantino, en buena parte porque se trataba de competir con los mismos productos, por ejemplo las pasas o los frutos secos. La novedad en el tránsito a los tiempos modernos radica en la agregación económica y cultural que Alicante realizo con el mundo anglogermano y que será fundamental en el desarrollo y prosperidad de la ciudad en el futuro.

Desde mediados del siglo XV los extranjeros se sintieron particularmente atraidos por las buenas perspectivas que para los negocios ofrecian las comarcas meridionales valencianas. Desde cuándo Alicante es una plaza interesante para los extranjeros, o iqué importancia tuvieron éstos en el comercio exterior o cuáles eran las colonias más importantes? son preguntas de difícil respuesta en el momento presente por la escasez de fuentes.

La impresión general es que, pese al incremento del tráfico portuario, la colonia extranjera en Alicante a fines del Medievo aún no era muy numerosa, siendo los operadores italianos los más numerosos, aunque buena parte de los negocios se segulan haciendo desde Valencia, como la compra de pasa, almendra, etc. que luego se embarcaba en Alicante por merca- 
deres locales o valencianos, que actúan como consignatarios de los italianos.

La importancia adquirida por la ruta de Flandes explica la presencia en Alicante de mercaderes de los países atlánticos, ingleses o flamencos, aunque a fines de siglo todavía lo hacen de forma esporádica.

Los que si demostraron un contínuo interés por los productos alicantinos fueron los alemanes. Durante el último cuarto de siglo la presencia de operadores alemanes en Valencia fue una realidad en aumento, plasmada en la actividad de dos importantes compañfas: la "Grosse Ravensburger", o Gran Companyia, y la "Xica Companyia", gestionada por los Ompis y los Ancarita (Ankereute) respectivamente, aunque siempre fue la de los Ompis la que acaparo el grueso de las actividades de los alemanes en Valencia. El interés de los alemanes en el reino de Valencia abarcaba muy diversos campos, desde el propiamente comercial, con operaciones de importacion-exportación de los más variados productos, sobre todo lombardos, o la venta al detalle en la ciudad de Valencia, al negocio del azúcar en la Safor o los esclavos. En el espacio meridional valenciano, aparte del azúcar citado, en tierras alicantinas los mercaderes del área anglogermana encontraban una serie de productos particularmente atractivos para la exportacion: higos, pasas, vino, azafrán, etc. Ya en 1468 vemos como la Gran Companyia tenía un factor desplazado en Alicante: Joan Alamany, que enviaba especias a Flandes en la nave del veneciano micer Jacobo Rinaldo. Kellebenz nos da la noticia de que los Ompis tenfan como delegado en Alicante a Antoni Mingot, que les compraba alazor, comino, anis, cera y grana.

En 1490 operaban en nuestra ciudad las dos compañfas alemanas, realizando transacciones con Flandes. El viajero alemán Jerónimo Múnzer encontro en su estancia en 1494 a Jos Schedler de Kenopten, que desde hacia muchos años ejercía como factor de la Compañia de los Ompis, aunque Schulte lo hace factor de los Ankereute.

Si en Valencia el grueso del comercio de los alemanes estaba controlado por los Umpis, aqui, en Alicante, parece suceder lo contrario, al menos en el tráfico con Flandes, donde et volumen de operaciones de los Ankereute en 1490 supera claramente a los Umpis.

A partir de los años setenta de siglo Alicante da un gran salto en su economía y pasa a insertarse en un espacio mucho más amplio que el de la Mancha mediterránea, cual es el del mundo anglogermánico, plasmado en la ruta de Flandes. Es una forma más de integración económica en la Europa de la época y más que de dependencia colonial o de periferia de una 
economía-mundo habría que hablar de división del trabajo, de interdependencia económica. Se trata de un itinerario con entidad propia y marcada personalidad en el momento del tránsito a la Modernidad, como lo demuestra la singularización que del mismo se hace en las fuentes, al indicarse Les naus de Flandes, precisión terminológica que abarca también al tipo de embarcación utilizada, la nave, de alto porte, ideal para estos intercambios que hacen de Alicante un puerto clave en el Mediterráneo meridional en la ruta que desde el Mediterráneo llevaba a los puertos atlánticos, en particular Flandes. Este comercio bilateral, en manos sobre todo de la oligarquía alicantina, tiene como base productos altamente especulativos del agro alicantino, como las pasas, el vino (en 1485 van a Flandes 918 botas, 6.000 cántaros y 2 pipas), los higos y los frutos secos, además del esparto y las manufacturas elaboradas con estas fibras. Era también un comercio de alto rendimiento fiscal para la Corona, el más importante del de larga distancia y una de las bases de la prosperidad de Alicante, que en 1490 alcanza la categoría de ciudad, en reconocimiento a su desarrollo económico y su ayuda a la Corona ${ }^{35}$.

En el reino murciano no he encontrado noticias de este tráfico con Flandes, aunque cabe presuponer su existencia por el puerto de Cartagena, mencionándose la importación de paños de Flandes, pero Italia sigue acaparando el grueso de las importaciones murcianas.

También en Málaga la presencia de mercaderes flamencos se detecta desde los momentos inmediatamente posteriores a la conquista cristiana, aunque son pocos y su interés parece centrarse en el comercio de pasas. Su actividad, como señala $\mathbf{M}^{2}$. T. López Beltrán, apenas tiene incidencia en el comercio portuario malagueño.

\section{AgRegación cultural al espacio angloeuropeo}

En el terreno cultural vemos como los humanistas multiplicaron su influencia a través de la imprenta, que sirvió para difundir verdades, errores o supersticiones, para empezar a crear una opinión pública mediante panfletos y propaganda. Incluso en medio de una población en su mayoría

\footnotetext{
${ }^{35} \mathrm{~A}$ mis ya citados trabajos sobre las relaciones entre Valencia y Flandes o el movimiento portuario en Alicante en la segunda mitad del siglo XV hay que añadir el de J.M. DEL ESTAL, Alicante, de villa a ciudad, Alicante, 1990.
} 
analfabeta, estos años se caracterizan por el peso principal de lo escrito y de la cultura intelectual sobre la anterior cultura oral. El modelo de hombre será el "hombre de los libros", el nuevo profesional. Por ello es inevitable que nos refiramos a la imprenta en este espacio mediterráneo, precisamente porque es un lazo de unión con el mundo germano.

La imprenta es el gran.vehículo que impulsó la difusión de las ideas humanistas y de sus logros cientificos y técnicos. Aunque su invento puede fecharse alrededor de 1450, es a finales de este siglo XV cuando se ha perfeccionado, permitiendo imprimir los textos literarios, filosoficos y filológicos emblemáticos de la nueva cultura.

Su difusión fue muy rápida por toda Europa. Desde las ciudades alemanas de Maguncia, Colonia, Augsburgo, etc. se extiende por Francia, Italia, España. En nuestra península no es casualidad que sea Valencia la primera plaza donde se imprimen libros y que su introducción en 1474 se deba al mercader alemán Jacobo Vitzlant, agente o asociado de la Compañfa de Ravensburgo, quien hizo venir al flamenco Lambert Palmart y creo el primer negocio editorial valenciano. El tema ha sido muy bien estudiado por Philippe Berger recientemente y no vamos a insistir en ello ${ }^{36}$. Pero nos sirve para recordar la agregación cultural que se produjo entre la ciudad de Valencia y Flandes-Alemania. Tipografos, impresores, libreros, como Palmart, Spindeler, Rosenbach, Kofman, Rix de Cura, etc. dejaron testimonio de su arte en nuestra ciudad y del intenso flujo humano y cultural que la imprenta trajo a Valencia. Tampoco es casualidad que cuando Vives se exilie de su ciudad natal lo haga a Brujas, centro intelectual europeo del momento.

Es interesante señalar que la imprenta no circula sólo en dirección Norte-Sur, sino que la interrelación cultural hispano-italiana es también muy fuerte. Las tipografías que traen los primeros impresores no son de procedencia germánica, goticas, sino la redonda italiana, mientras que un español, el cardenal Juan de Torquemada, es el primer autor que publica un libro en la Ciudad Eterna, y es de todos conocido la gran cantidad de autores hispanos que publican en Roma o Venecia.

Pero una cosa es Valencia, la capital del reino, cuyo potencial en todos los ordenes no admite comparación con el resto del territorio, y otra el resto del reino. Valencia monopolizó el nuevo arte tipográfico durante

${ }^{36}$ PH. BERGER, Libro y lectura en la Valencia del Renacimiento, Valencia, 1987. 
más de cien años, pues hasta los albores del siglo XVII no aparecen talleres en otras ciudades del país, en Orihuela en 1602 y en Alicante nada menos que en 1689.

En Murcia sucede exactamente lo mismo y no será hasta fines del Quinientos en que se cree una oficina de imprenta en la capital. De otras ciudades del Mediterráneo meridional no he encontrado datos, pero todo apunta a una fecha tardía para la instalación de la imprenta.

En Andalucia fue Sevilla el quinto lugar donde se estableció la imprenta, mientras que respecto a la producción de libros y hojas volantes durante el periodo incunable le corresponde el segundo lugar 'entre las imprentas más activas (130), mientras que en Valencia sólo se imprimen 82 libros. Señalemos también que, por primera vez en la península, son nativos sus prototipógrafos, además de predominar la letra gótica sobre la redonda.

En definitiva, son los centros más dinámicos desde el punto de vista mercantil, los que también aparecen en la avanzada de la cultura impresa: Valencia, Sevilla, Barcelona, Zaragoza... ${ }^{37}$.

En el campo de las artes, si uno de los objetivos básicos del Renacimiento se centra en la representación fidedigna de la realidad (el hombre y el espacio que le rodea), hay que convenir con Panofsky que además del renacimiento italiano se produce otro, y muy destacado también, en los Paises Bajos, representado por los Van Eyck, Maestro de Flemalle, Roger van der Weiden. La pintura valenciana de la segunda mitad del siglo XV nos demuestra que el Renacimiento en versión flamenquizante de los Dalmau, mestre de Bonastre, etc. se oponen a los pintores aferrados a los presupuestos tardomedievales. La segunda mitad del siglo XV se caracteriza en Valencia por la fuerte influencia de la pintura flamenca introducida desde el final de la década de los años treinta. Frente al grácil refinamiento y exquisita estilización del estilo internacional, ahora se tenderá, como señala Daniel Benito Goerlich, a unas nuevas formas apoyadas en la sensibilidad hacia lo inmediato e individual y a una nueva captación del espacio al que no son ajenas las nuevas conquistas de la perspectiva ${ }^{38}$. Este conocimiento directo de la pintura de Flandes

\footnotetext{
${ }^{37}$ VV.AA., Historia de la imprenta hispana, Madrid, 1982; VV. AA., La imprenta valenciana, Valencia, 1990.

${ }^{38}$ D. BENITO, Valencia y Murcia, col. La España Gótica, IV, Madrid, 1989.
} 
fue posible para los artistas valencianos, dado que Jan Van Eyck recorrió la península en 1428 y Alfonso V compró obras suyas en 1444. Desde estas fechas y hasta finales de siglo hay testimonios de pinturas y artistas flamencis en Valencia que influirían de manera decisiva en la producción artística local. Recordemos la espléndida tabla del Juicio final de Van de Stockt, adquirida por los jurados en 1494 para la Casa de la ciudad. O el paso por la ciudad en 1470 del cordobés Bartolomé Bermejo, que reforzó el influjo flamenco.

Valencia se erige en pionera de la recepción de los dos Renacimientos, el italiano y el flamenco, siendo en la pintura en donde más destaca el arte valenciano del Renacimiento, con nombres como Rodrigo de Osona, Pablo de San Leocadio, Yánez de la Almedina, Vicent Macip, etc. Una pintura de temática básicamente religiosa, hasta que a partir de la década de 1470 , por la incorporación de la pintura ferraresa a Valencia o por la presencia de escultores venidos de Alemania y de los Paises Bajos, se puede decir que el sentido religioso del arte cambia al convertir los temas, hasta entonces exclusivamente religiosos, en pretexto para plasmar la realidad e incorporar el principio de la individualidad y algunas recuperaciones arqueológico-mitológicas también venidas de fuera y que se desarrollarán plenamente en el s. XVI. Valencia, por tanto, se convierte una vez más en la encrucijada donde se sitentizan las esencias vaneyckianas y leonardes$\operatorname{cas}^{39}$.

Ahora bien, la posición de Valencia como foco artístico de primer orden no resiste la comparación con otros núcleos del sur del Mediterráneo, y si aqui encontramos una huella del arte flamenco, a través de la pintura, en el resto del espacio anal izado la presencia italiana, a través del Renacimiento, irrumpe con tal fuerza, es tan clara, que anula cualquier posibilidad de agregación cultural a otro espacio europeo, en este caso el flamenco. Sencillamente, no existe. Murcia y las costas granadinas se abren receptoras culturalmente al Mediterráneo, no al Atlántico. Los artistas, las piezas se traen de Italia, no de Flandes. El viejo mar todavía sigue siendo un vehículo cultural de primer orden.

\footnotetext{
${ }^{39} \mathrm{X}$. COMPanY, La pintura del Renaiximent (al Pais Valencià), Valencia, 1987; .4. JoSÉ I PitarCh, Historia de l'Art Valencià, Valencia, 1986.
} 


\section{CONCLUSIONES}

Así pues, en estos años de transición de los tiempos medievales a los modernos se vive en los ámbitos cultos la euforia de un mundo en movimiento, explorador, navegante, curioso, mercader, inventor, pero al mismo tiempo firmemente asentado en sí mismo. Transición, crisis desde luego ante el cambio paulatino de imagen y de valores que se está sufriendo, pero apuesta en el fondo optimista por un presente y futuro que los hombres mismos, en su existencia histórica y concreta, pueden moldear.

Pero esta circulación de hombres, mercanclas y culturas se mueve a un tempo diferente según el espacio mediterráneo de que se trate, y si en Alicante a finales del siglo XV los contactos con la Europa anglogermánica desempeñan un papel importante, en cambio no puede decirse lo mismo de Málaga, donde son escasos, y donde la huella nazarı sigue siendo fuerte y hace que siga mirando sobre todo hacia el Mediterráneo, hacia Italia más que hacia Flandes.

\section{RÉSUMÉ}

A la fin du Moyen Age, dans la Méditerranée Sud-Occidentale, convergent des routes, des gens, des marchandises, des nefs, des idées et des techniques novatrices qui, peu à peu, se diffusent à travers ses États riverains avec une intensité inégale. Ses terres, qui sont toujours des frontières, surtout maritime (pirates et corsaires), et ses villes portuaires (comme Alicante) se revitalisent avec le commerce des pays atlantiques (Flandres) et la présence d'opérateurs économiques italiens et allemands. Une intense circulation d'hommes (esclaves, arabes et juifs expulsés) et de cultures, depuis l'Islam à la Renaissance italienne, avec la découverte de l'imprimerie, nous montre un monde vivant et à la fois refermé sur lui-même, qui continue à regarder davantage vers la Méditerranée que vers l'Atlantique. 


\section{SUMMARY}

Towards the end of the Middle Ages, there is a convergence of routes, peoples, ships, merchandises and innovative ideas and techniques in the Southwestern Mediterranean that little by little begin to filter into the fabric of the coastal States although with uneven intensity. These are still borderlands, especially maritime coastal areas (pirates and privateers), whose port cities (such as Alicante) are revitalized by trade with Atlantic countries (Flanders) and the presence of Italian and German economic operatives. Intensive circulation of men (captives, exiled Jews and Moors) and cultures from Islam to the Italian Renaissance along with the advent of printing are all proof of a world full of vitality yet somehow anchored to itself, one which looks more towards the Mediterranean than towards the Atlantic. 